BNL- -37049

DE86 0012015

ETALDATIOA TECHIQUES FOR X-RAT MIRRORS AND STSTEMS USING VISIBLB LIGHT

\author{
P. Z. Takace and J. Colbert \\ Instrumentation DIviaion \\ Brookhaven National Laboratory \\ Opton, $\mathbf{N T}$ 11973-5000
}

Septenber 1985

\title{
DISCLAIMER
}

This report was prepared as an account of work sponsored by an agency of the United States Government. Neither the United States Government nor any agency thereof, nor any of their employees, makes any warranty, express or implied, or assumes any legal liability or responsibility for the accuracy, completeness, or usefulness of any information, apparatus, product, or process dixclowed, or represents that its use would not infringe privately owned rights. Reference berein to any specilic commercial product, proceses, or service by trade name, trademark, manufacturer, or otherwice does not neceasarily constitute or imply its endorsement, recommendation, or favoring by the United States Government or any agency thercof. The views and opinions of authors expresed herein do not necessarily state or reflect those of the United States Government or any asency thereor.

*This work was supported by the U. S. Department of Energy under Contract DB- $1 \mathrm{CO2}-76 \mathrm{CHOOO16}$.

By scceptance of this article, the publisher and/or recipient acknowledges the U. S. Government's right to retain a nonexclusive, rogalty-free license in and to any copjright covering this paper. 


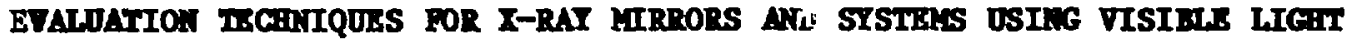

\author{
P. Z. Takacs and J. Colbert \\ Instrumentation Division \\ Brookhaven National Laboratory \\ Upton, NY 11973-5000
}

\begin{abstract}
Severely diffraction-broadened visible light images

from grazing incidence optical systems are measured and analyzed using a diffraction integral model to predict slope errors and image quality at XUV wavelengths.
\end{abstract}


Introduction

Optical systems designed to utilize extreme ultraviolet (EUV) and x-ray photons from synchrotron radiation (SR) light sources with grazing Incidence optics generally have entrance apertures that are long in the horizontal plane and narrow in the vertical plane. Apertures that are 1 mrad high by several milliradians wide et a distance of 10 meters from the source with a focal length of 1 meter are typtcal. A point source Illuminated by a red He-Ne laser beam Inaged through a system with this aperture and focn i length results in a $1.2 \mathrm{~mm}$ high image that is severely broadened by diffractinn. Component alignment and wavefront quality assessment with visiule light is difficult when the diffraction limit is 80 severe. The nie of vistble light for system alignment 1s, however, a necessity, because alignment under actual operating conditions and at operating wavelengths in ultra high vacuum (UHV) chambers is totally impractical. Except in rare instances, UHV components are not accessible for alignment adjustments. How, then, can we make use of the information available in the severely diffraction-limited visible image to assess the perforwance of our system at $x$-ray wavelengths?

Our approach to solving this problem is to make use of image quality inforwation that is contalned in the full diffraction pattern of the rectangular aperture, not only in the central maximum, but also in the shape of the higher order maxima. This method is particularly useful for calibrating mirror-bender mechanisms for bent-cylinder optics, since the amount of preload on the bending device is generally not known during the inftial alignment. We measure the point spread function (PSF) of the system by scanning across the image of a pinhole source with a narrow slit, and then wodel the results with a simple diffraction integral calculation that runs on an HP 9836 desktop computer. 
Mode1

The Intensity distribution in an ideal image limfted by a rectangular aperture is the well known (sinc) ${ }^{2}$ function. This is strictly true only in the Image plane. For points not in the Image plane, the intensity pattern for an ideal converging wavefront through a rectangular aperture was calculated analyt1cally by Thompson [1] In 1959. In the case of aberrated wavefronts, with which we are dealing, an analytic treatment that includes the entire volume of space near the geometric focus doeg not exist. For this reason we developed a simple one-dimensional Fresnel-Kirchhoff numerical integration program that runs in BASIC on an KP 9836 desktop computer. The physical Image formation problem is 1llustrated in Fig. 1. A wavefront emerging from the exit pupil of a system converges to form an image in an observation plane. The image formation process can be described by the Fresnel-Kirchhoff scalar diffraction integral formulation for the amplitude of the fleld in the observation plane:

$$
U(P)=\frac{1}{1 \lambda} \iint_{A} U(Q) \frac{e^{1 k r_{P Q}}}{r_{P Q}} d A
$$

where the Integration is carried out over the exit pupil area, $r_{P Q}$ is the distance between a point In the exit pupil and the observation point, and $U(Q)$ describes the amplitude and thase of the wavefront in the aperture. Since we are dealing with a narrow rectangular aperture and a narrow slit detector, we can treat the problem as a one-dinensional integral over the width of the aperture. Since we are only interested in relative amplitucies, we may neglect the constant terms outside the integral in Eq. (1) and, with reference to Fig. 2, we may rewrite the diffraction integral as: 


$$
U\left(P_{f}\right)=\int_{-\frac{W}{2}}^{\frac{W}{2}} e^{1 t_{F}\left(Q_{1}\right)} e^{1-a b\left(Q_{1}\right)} e^{1 k\left[z_{o}^{2}+\left(P_{j}-Q_{1}\right)^{2}\right]^{\frac{1}{2}}} d Q_{1}
$$

where the distance $r_{P Q}$ is written explicitly in term of the coordinates of the source and observation points. The aperture function is written as the product of two terms: $F$ is the phase of the Ideal converging wavefront;

$\Phi_{a b}$ is the phase variation of the aberration term. The focus term is simple the sag of the 1deal wavefront in the aperture, approximated as

$$
\phi_{F}\left(Q_{1}\right)=\frac{2 \pi}{\lambda} \frac{Q_{1}{ }^{2}}{2 F}
$$

The aberration term depends on the particular situation under consideration. We have found that in most cases it is sufficient to model the aberration as a single period sinusold, whose period is the full aperture width, $W$, with two free parameters: the amplitude, A, and the phase, $\Phi_{0}$ :

$$
\phi_{a b}\left(Q_{1}\right)=A \sin \left(\frac{2 \pi Q_{1}}{W}+\Phi_{0}\right)
$$

The physical justification for this function is that it approximates, to lowest order in one dimension, the type of figure defect one would expect in polishing long, cylindrical aspheres: namely a surface with a low spot either in the middle or neer an end. When appropriate, a more detalled aberration function can be used, as in the case of a known gravity sag correction ss shown below. The form of Eq. (2), using the wavefront terms as in Eqs. (3) and (4), is suited for direct numerical integration. The aperture is divided into Intervals based upon the smallest Fresnel zone width, and for each observation point, $P_{j}$, the integral is summed over 
each aperture point, taking care to separate the real and imaginary parta. The iutensity, which is the observable quantity, is the square modulus of the amplitude.

\section{PGM Studies}

The Inttial Imjëtus for this analysis came from the desire to understand more fully the nature of zero-order 1mages produced by the NSLS Plane Grating Monochromator (PGM) [2]. A set of recent zero-order scans from the U4 PAM is shown In FIg. 3. In this case the source was the actual SR source, monochromatized through a narrow band interference f11ter centered at $405 \mathrm{~nm}$. At this wavelength the source 18 still diffraction IImited and the tmages represent the actual PSF of the instrument. None of the 1mages exhibits the Ideal (sinc) ${ }^{2}$ form. By Inserting a plane mirror Into the PGM after the P5 collimating mirror, we were able to make PSF measurements on the P5 mirror alone by autocollimating the light from a He-Ne laser point source. The results are shown in the upper half of Fig. 4. The model aberration function that best-fits the data is one with an amplitude of $0.1 \lambda(63 \mathrm{~nm})$ and a phase of $\frac{\pi}{2}$. Physically, this is equivalent to a symmetric cosine function which suggests a surface with a low spot in the center (or a high spot). The effect of this model is to raise the first order maxima on each side of the central peak and fill in the first order minima on each side. The equivalent single-pass RMS wavefront slope error calculated from this aberration function is 2.7 arc seconds.

The lower part of Fig. 4 illustrates the type of aberration seen with the P3 focussing mirror installed in the system. In this case measurements were made with a He-Cd laser and pinhole source. The model aberration function that best-fits this observation is one with an amplitude of 
$0.1 \lambda(44 \mathrm{~nm})$ wth no phase offaet. This aberration 1s equivalent to a sine function with an RMS slope error of 3.8 arc seconds. This function also has a net tilt, which serves to direct most of the aberiated energy Into the right-hand first-order peak at the expense of the left-hand peak. Th1s model behavior reproduces the actual data observation quite well. The usefulness of this RMS slope error measurement lies in the fact that 1t allows us to estimate the performance of the PGM at shorter wavelengths. At shorter wavelengths, the actual SR source acts as an Incoherent source over the aperture of the PGM. The observed 1mage is the convolution of the Ideal source-size-limited (SSL) Image with the slope error function. In this case the SSL Image for P3 is on the order of $20 \mu \mathrm{m}$ wide at FWHM, while the 3.8 arc second slope error results in an Image smearing of about $19 \mu \mathrm{m}$. The SSL is thus matched exactly to the aberration in the system. One consequence of this observation is that the system performance cannot be improved by reducing the size of the source. The quality of the optical components would need to be improved before a reduction in source size would be useful.

\section{Bent Mirror Studies}

The PSF analysis technique is also useful for assessing the quality of bent cylinder mirrors and callbrating mirror bender mechanisms. A serles of measurements and models is shown in F1g, 5 for the $X-14$ bent mirror [3] Illuminated by a He-Ne laser and pinhole source. This mirror is $62 \mathrm{~cm}$ long, of which the central $50 \mathrm{~cm}$ is illuminated at a grazing angle of 10 mrads. The fonal length is nominally 10 meters, which is where the scanning detector was located. By driving the stepping motor on the bending mechanism first to the best focus and then beyond to an overbent 
condition, one can mudel the observed Image scans as shown In F1g. 6 . For the sase of the best image an aberration function is required that consists of two terms: one accounts for the gravity-induced sag along the length of the surface, the other is a residual sinusold to account for the remainder of the observed PSF averration. The amount of residual sinusoid required has an Emplitude of $19 \mathrm{~nm}(0.03 \lambda$ at $632.8 \mathrm{~nm})$ in the aperture, which produces a 3.4 arc second wavefront slope error. When the mirror is overbent by 200 steps, the value of the focal length parameter in equation (3) changes from $10.0 \mathrm{~m}$ to $5.80 \mathrm{~m}$, and for 300 steps the focal length becomes $4.50 \mathrm{~m}$. The agreement between model and data is quite remarkable. The sensitivity to the focal length parameter is such that changing the value from $4.50 \mathrm{~m}$ to $4.49 \mathrm{~m}$ reduces the left-hand peak by about $20 \%$ relative to the right-hand peak. The most striking feature of this analysis is that the aberration function described above is absolutely essential to reproduce the observed data.

\section{Sunmary}

This study has shown the usefulness of the PSF analysis technique for assessing the quality of grazing Incldence optical systems used with SR. The fact that most $S R$ optical systems have rectangular acceptance apertures simplifies the calculation of the diffraction integral model. The test is easy to set up and is convenient to perform with visible light sources. In some cases even the actual SR source can be used because it acts as a coherent, diffraction-limited source for small apertur? SR optics. Folding mirrors and vacuum windows do not appear to have a significant effect on the observed images; most of the residual aberrations are inherent in the surfaces observed to date. For a good signal-to-noise ratio, one must be 
able to ahield the photomultiplier detector from stray room light by restricting the field of view and by restricting the band pass to the laser wavelength. The model is easy to program and runs rapidly on an $\mathrm{HP} 9836$ desktcp conputer, provided the aperture size is not too large. One quickly can get a feel for the effect of various types of aberrations on the observed intensity patterns.

Acknowledgements

The authors wish to express their appreclation to Cullie Sparks, Gene Ice and Tony Habenschuss for making the measurements on the $X-14$ beam 11ne. The assistance of Francesco Sette and Christiano Capasso in making measurements on the U4 PGM is also acknowledged. 


\section{REFERENCES}

[1] B.J. Thompson, J.0.S.A. 73 (1959) 905.

[2] M.R. Howe11s, Nucl. Instrum. \& Methods 177 (1980) 127-139.

[3] Gene Ice, private communication. 
FIGURE CAPTIONS

Figure 1. Schematic diagram of the Image formation process. A pln-hole source produces an 1deal spherical wave Inctdent on a grazing Incldence component or system. Emerging aberrated wavefront converges to a focus in the Image plane where a scanning slit and detector record the one-dimensional point spread function. Figure 2. Geometry of the diffraction integral model. For each point, $P_{j}$, In the observation plane, the optical path contribution from all points, $Q_{1}$, in the aperture is computed and summed.

F1gure 3. Plots of the measured zero order images from the U4 plane grating monochromator, taken with the SR beam as the source, using a narrowband $405 \mathrm{~nm}$ wavelength f1lter. The four ranges are designated from most grazing to least grazing Inctdence as: $---=\mathrm{P} 1, \cdots \ldots+\mathrm{P} 2, \ldots \ldots \ldots=\mathrm{P} 3, \square=\mathrm{P} 4$. The FWHM depends mainly on the effective aperture width of each range; the asymmetry and distortion in the higher order diffraction maxima depend on the type of aberration in the system. 


\section{FIGURE CAPTIONS (continued)}

Figure 4. (Upper) - Image produced by the P5 collimating parabolotd a.lone in an autocollimation geometry. Model (dashed line) aberration function is a single period cosine function with a $63 \mathrm{~nm}$ amplitude, which indicates that the mirror produces a single-pass RMS wavefront slope error of 2.7 arc seconds. (Lower) - Solid line is Image from the P3 system. Dashed ilne 1s the mode1 and dotted line is the 1deal PSF for th1s aperture. Model Indicates an asymmetric sine function aberration with smplitude of $44 \mathrm{~nm}$ and an RMS wavefront slope error of 3.8 arc seconds. The asymmetric sine function has net tilt across the surface, which pushes more intensity into the right side higherorder maxima than Into the left.

Figure 5. A serles of scans (solid 11nes) from the bent mirror on $x-14$ as a function of relative stepping motor drive setting. The model curves (dotted lines) Include effects of a gravity-Induced mirror sag and a residual sinusoidal aberration with a 3.4 arc second RMS slope error. 


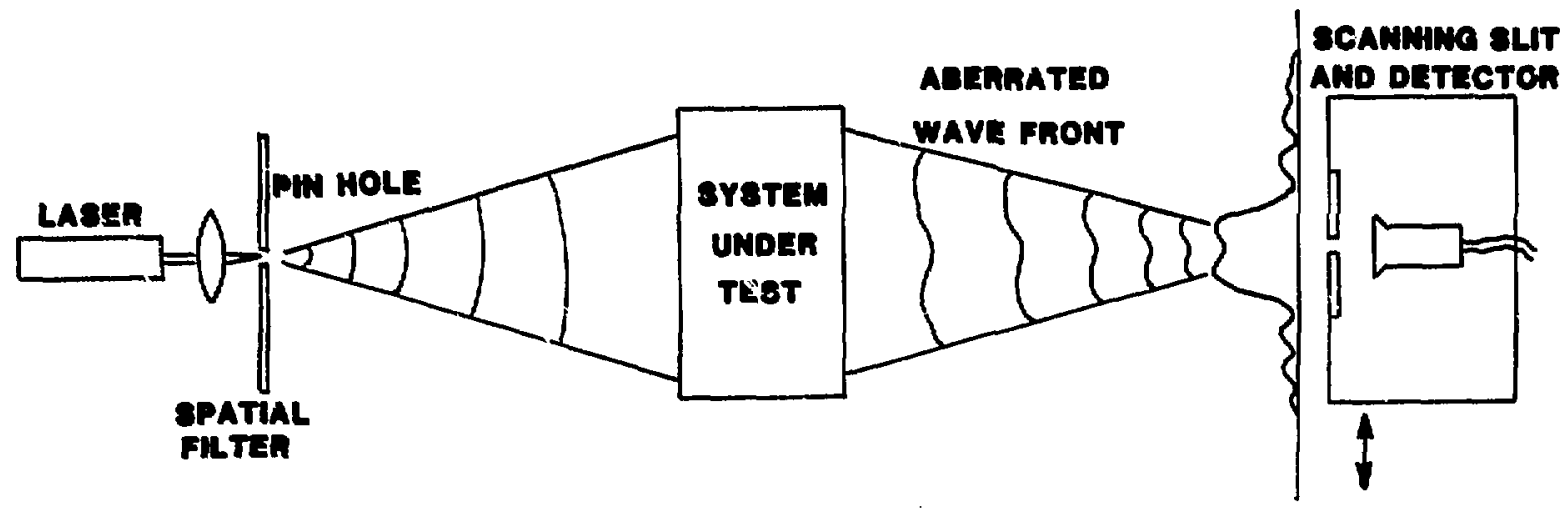

Figure 1 
I

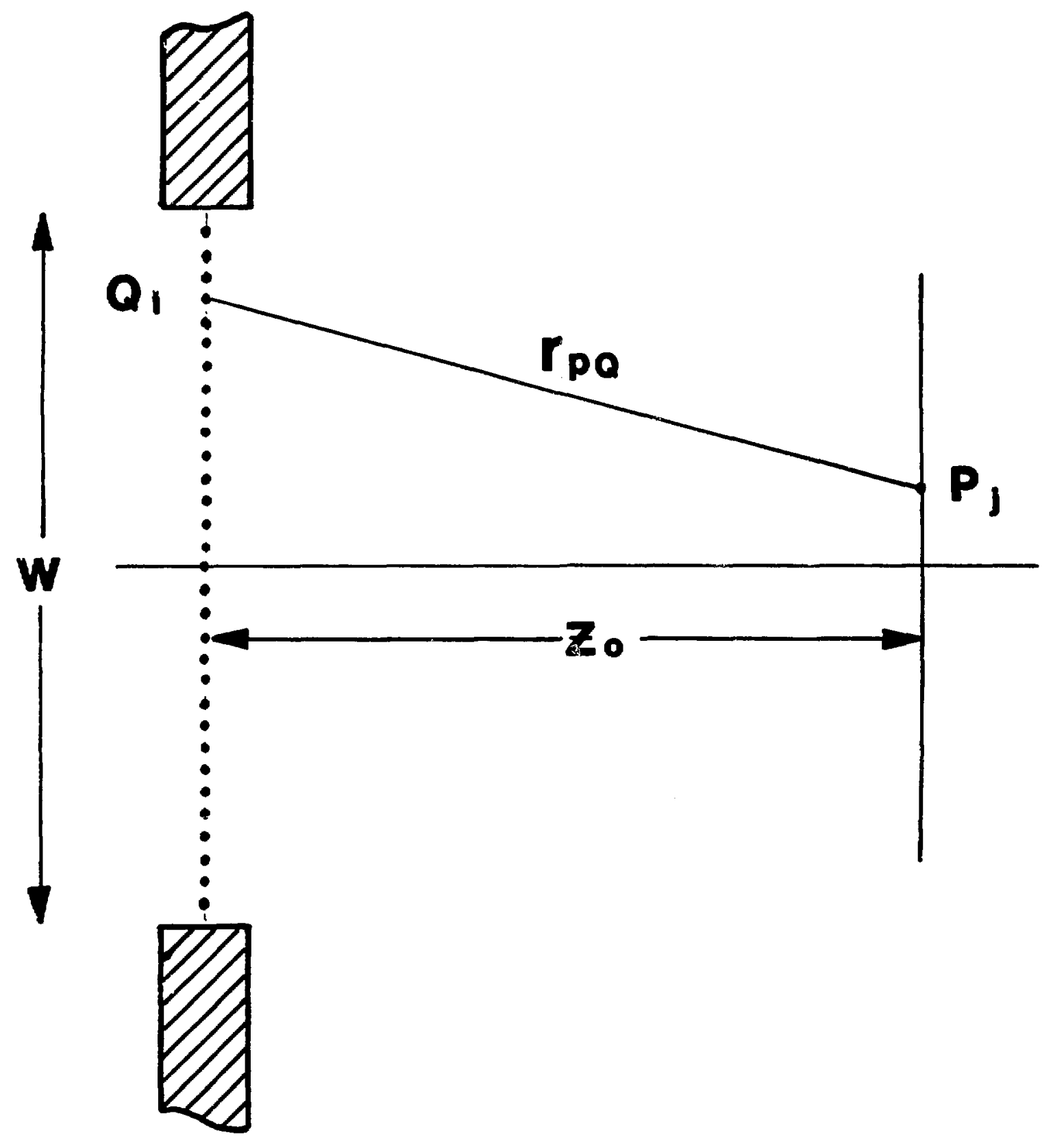

Ftgure 2 
U4 PGM Zero Order Images

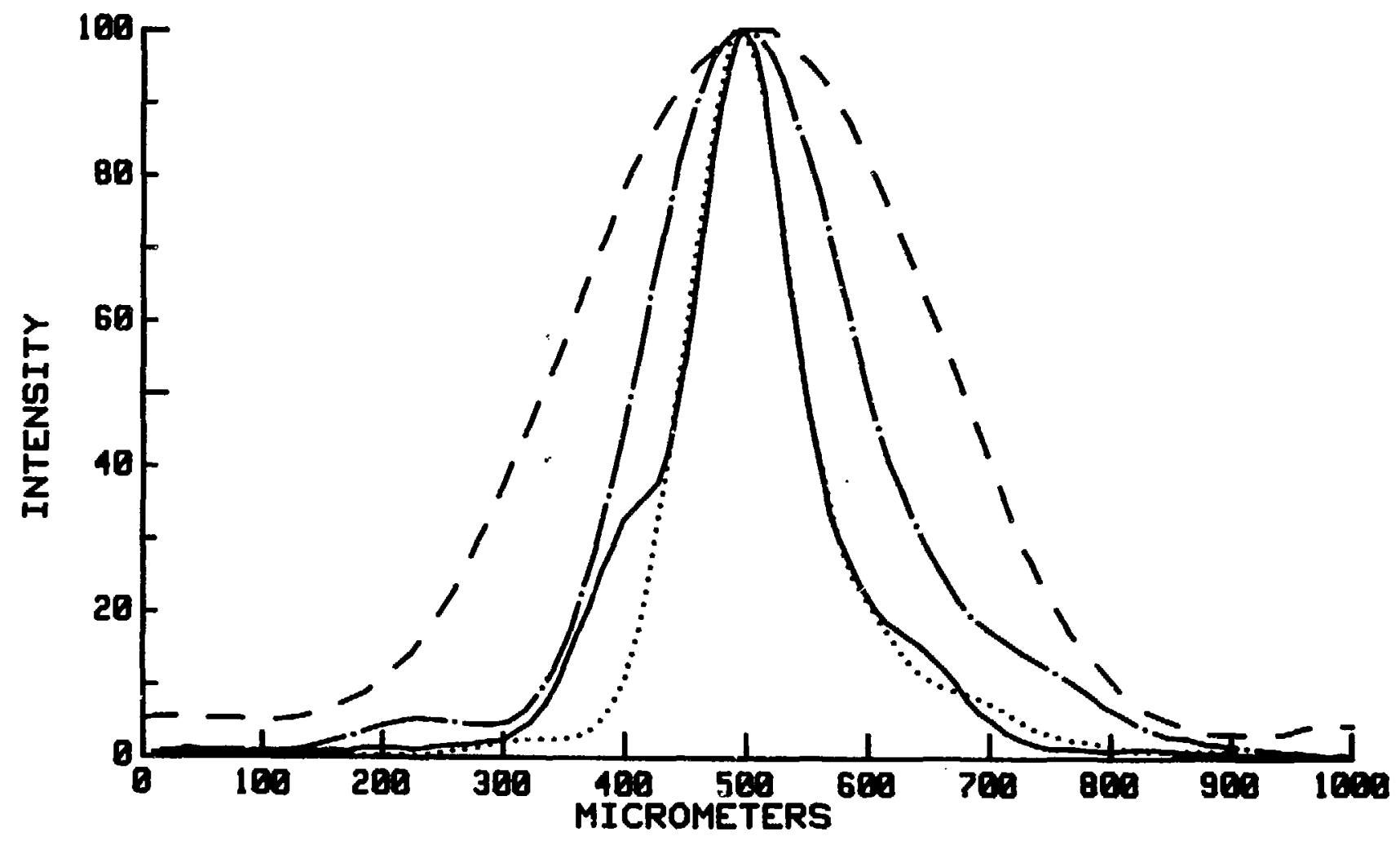


U4 PGM: P5 alone

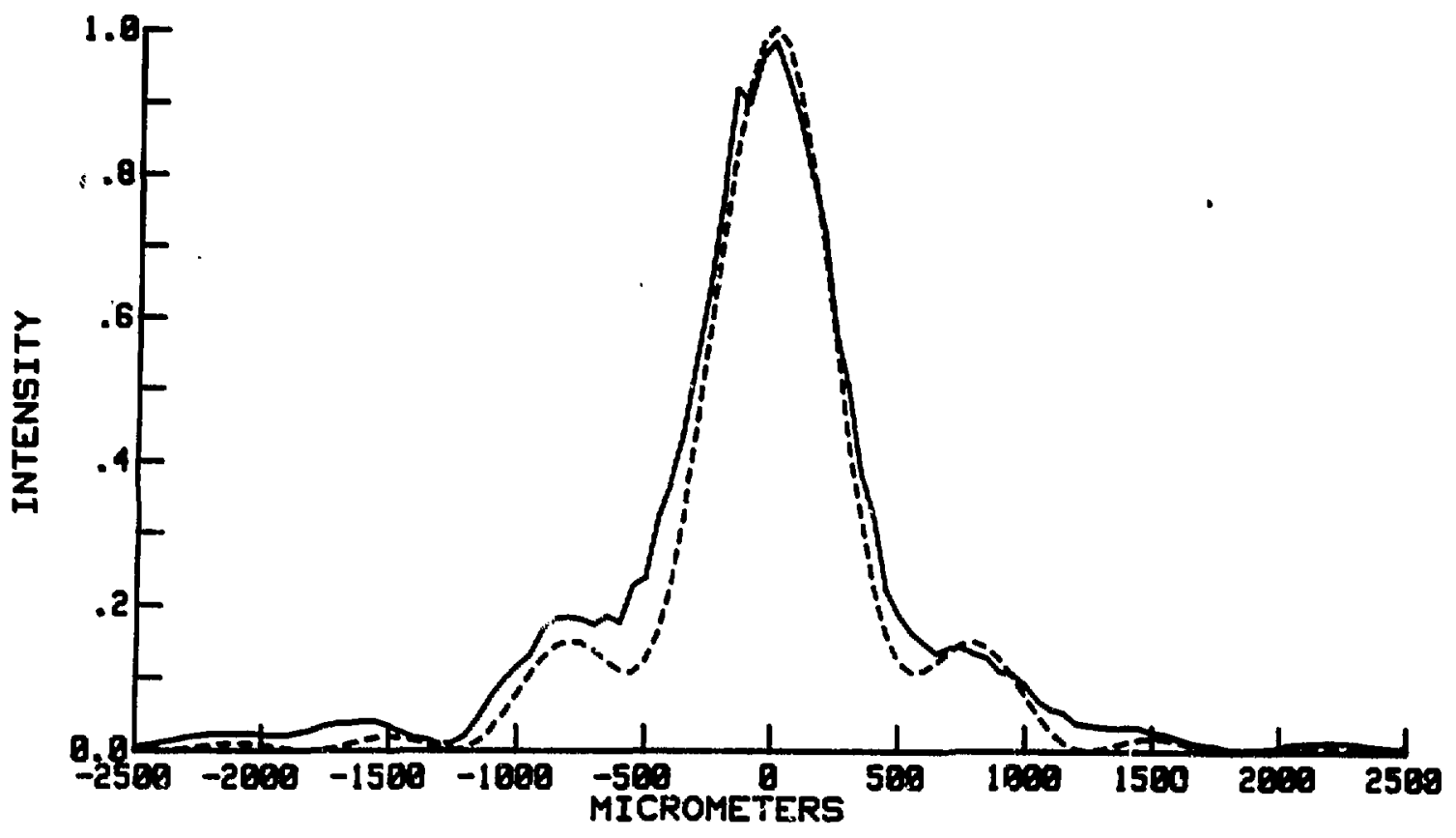

U4 PGM: P3 system

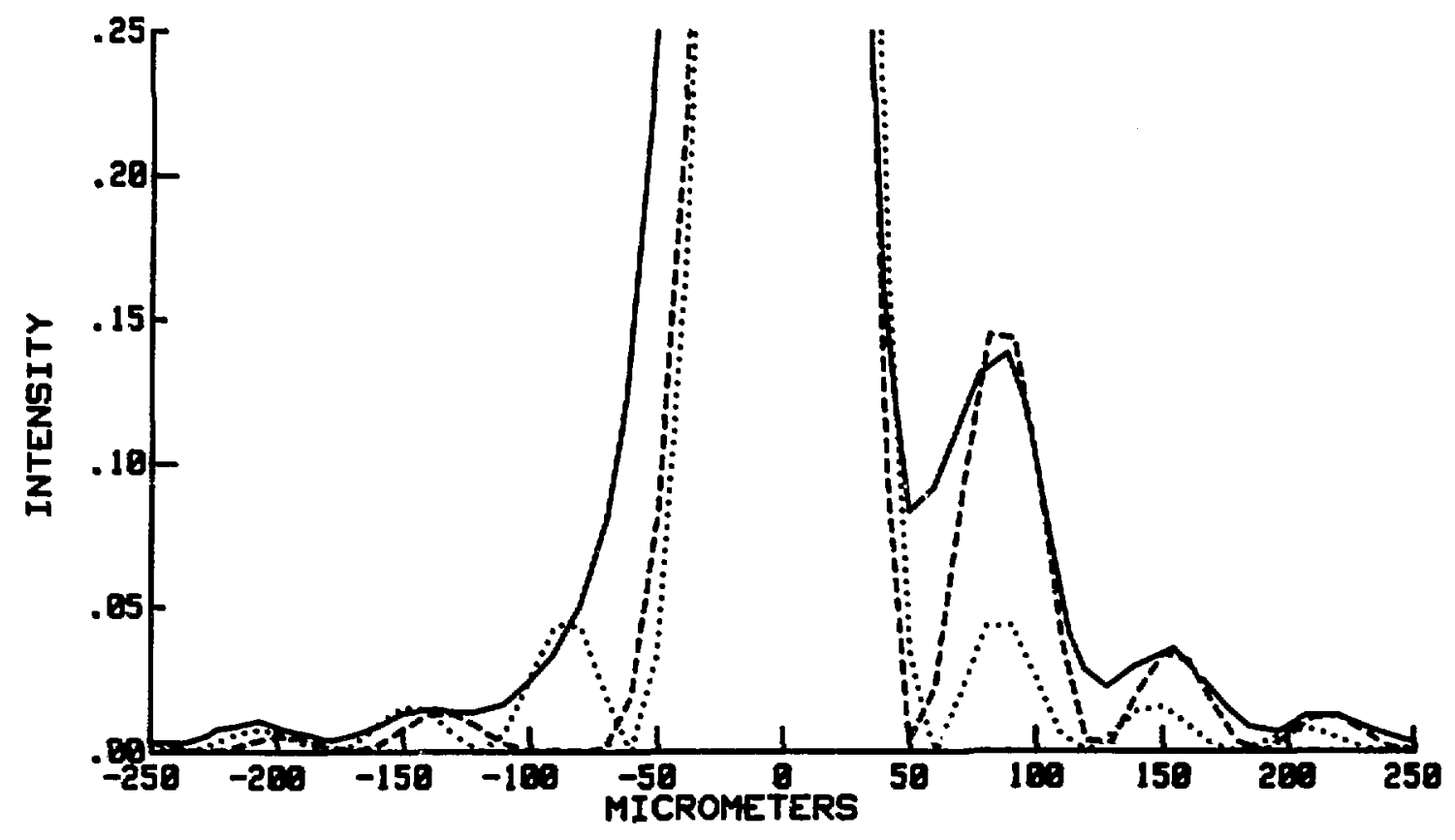




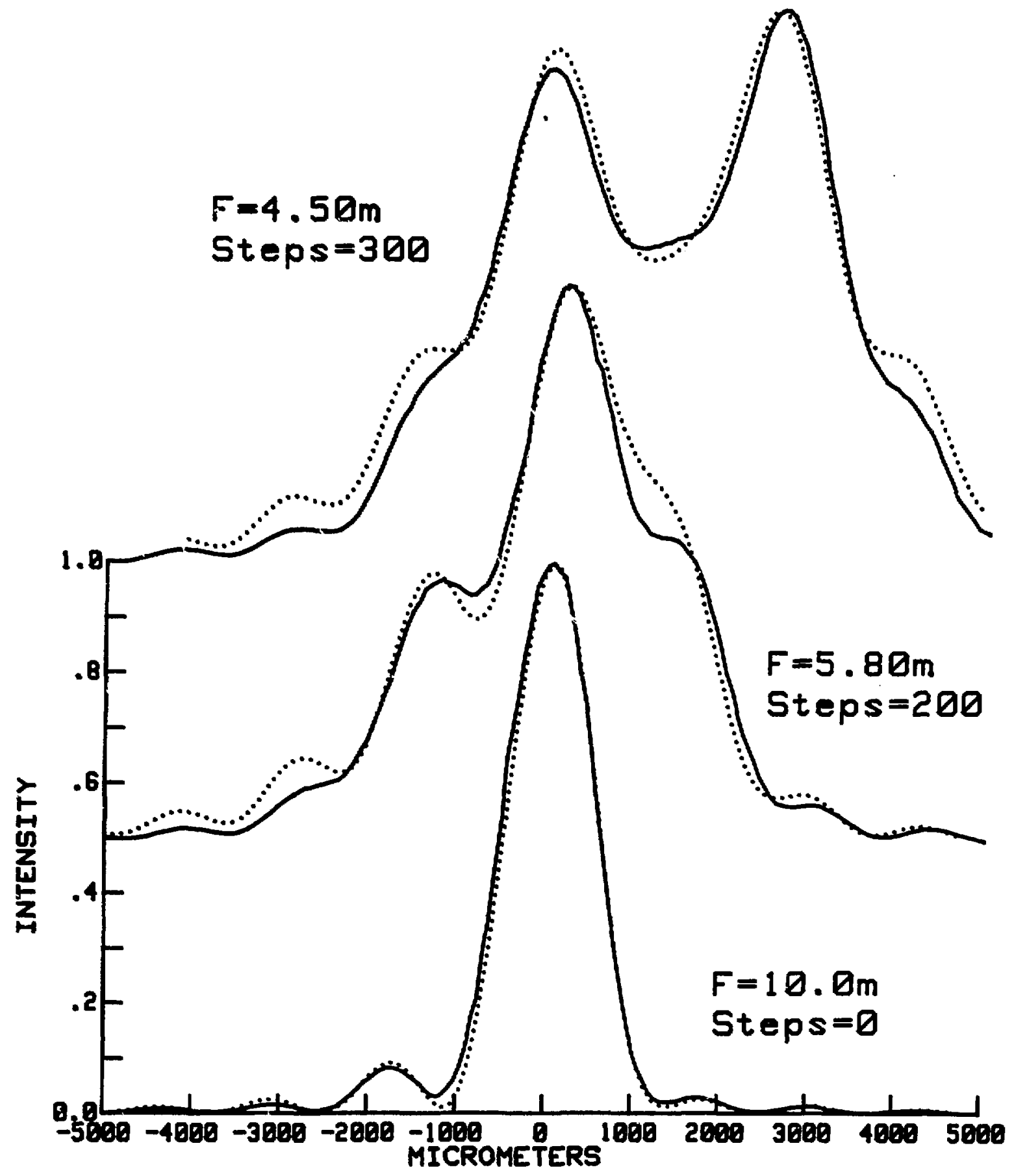

Figure 5 